\title{
Autoregressive single-step test-day model for genomic evaluations of Portuguese Holstein cattle
}

\author{
A. A. Silva, ${ }^{1}$ D. A. Silva, ${ }^{1}$ F. F. Silva, ${ }^{1}$ C. N. Costa, ${ }^{2}$ P. S. Lopes, ${ }^{1}$ A. R. Caetano, ${ }^{3}$ G. Thompson, ${ }^{4,5}$ \\ and J. Carvalheira ${ }^{4,5 *}$ \\ ${ }^{1}$ Departement of Animal Science, Federal University of Viçosa, Viçosa 36570-000, Brazil \\ ${ }^{2}$ Embrapa Dairy Cattle, Juiz de Fora 36.038-330, Brazil \\ ${ }^{3}$ Embrapa Genetic Resources \& Biotechnology, Brasília 70770-901, Brazil \\ ${ }^{4}$ Research Center in Biodiversity and Genetic Resources (CIBIO-InBio), University of Porto, Vairão 4485-661, Portugal \\ ${ }^{5}$ Institute of Biomedical Sciences Abel Salazar (ICBAS), University of Porto, Porto 4050-313, Portugal
}

\section{ABSTRACT}

The multiple-lactation autoregressive test-day (AR) model is the adopted model for the national genetic evaluation of dairy cattle in Portugal. Under this model, animals' permanent environment effects are assumed to follow a first-order autoregressive process over the long (auto-correlations between parities) and short (autocorrelations between test-days within lactation) terms. Given the relevance of genomic prediction in dairy cattle, it is essential to include marker information in national genetic evaluations. In this context, we aimed to evaluate the feasibility of applying the single-step genomic (G)BLUP to analyze milk yield using the AR model in Portuguese Holstein cattle. In total, 11,434,294 test-day records from the first 3 lactations collected between 1994 and 2017 and 1,071 genotyped bulls were used in this study. Rank correlations and differences in reliability among bulls were used to compare the performance of the traditional (A-AR) and single-step (H-AR) models. These 2 modeling approaches were also applied to reduced data sets with records truncated after 2012 (deleting daughters of tested bulls) to evaluate the predictive ability of the H-AR. Validation scenarios were proposed, taking into account young and proven bulls. Average EBV reliabilities, empirical reliabilities, and genetic trends predicted from the complete and reduced data sets were used to validate the genomic evaluation. Average EBV reliabilities for H-AR (AAR) using the complete data set were $0.52(0.16)$ and 0.72 (0.62) for genotyped bulls with no daughters and bulls with 1 to 9 daughters, respectively. These results showed an increase in EBV reliabilities of 0.10 to 0.36 when genomic information was included, corresponding to a reduction of up to $43 \%$ in prediction error

Received June 11, 2018.

Accepted January 27, 2019.

*Corresponding author: jgc3@cibio.up.pt variance. Considering the 3 validation scenarios, the inclusion of genomic information improved the average EBV reliability in the reduced data set, which ranged, on average, from 0.16 to 0.26 , indicating an increase in the predictive ability. Similarly, empirical reliability increased by up to 0.08 between validation tests. The $\mathrm{H}-\mathrm{AR}$ outperformed A-AR in terms of genetic trends when unproven genotyped bulls were included. The results suggest that the single-step GBLUP AR model is feasible and may be applied to national Portuguese genetic evaluations for milk yield.

Key words: autoregressive test-day model, Holstein cattle, single-step genomic BLUP

\section{INTRODUCTION}

Genomic selection has become a standard procedure in dairy cattle breeding given its potential to increase selection accuracy and to reduce the generation interval. One of the most used methods in genomic selection is based on the multistep procedure (VanRaden, 2008). In this procedure, the first step requires the estimation of traditional EBV. In the second step, daughter yield deviations (DYD) or deregressed phenotypes are calculated. In the third step, direct genomic values are predicted for genotyped animals using DYD or deregressed phenotypes as pseudo-phenotypes (VanRaden, 2008; Hayes et al., 2009; VanRaden et al., 2009). Finally, the blending of direct genomic values with parent averages (PA) is performed to yield genomic breeding values (GEBV; VanRaden et al., 2009). In general, this procedure has shown some disadvantages, such as the loss of information attributed to pseudo-phenotypes instead true phenotypes. Additionally, this approach is very complex and requires several approximations, which may reduce the accuracy and induce bias on the GEBV (Koivula et al., 2015).

The single-step genomic BLUP (ssGBLUP), where phenotypes, pedigree, and genotypes are jointly used 
to generate GEBV as a direct output, is a suitable alternative to the multistep method (Misztal et al., 2009; Aguilar et al., 2010; Christensen and Lund, 2010). In ssGBLUP, the traditional pedigree-based relationship matrix (A) is replaced by the $\mathbf{H}$ matrix, which integrates $\mathbf{A}$ and a genomic relationship matrix $(\mathbf{G})$. The ssGBLUP has been successfully applied to Holstein cattle (Lourenco et al., 2014; Koivula et al., 2015; Jattawa et al., 2016) and outperformed the multistep procedure.

In Portugal, the autoregressive test-day (AR) model for multiple lactations proposed by Carvalheira et al. (2002) has been routinely used for the national genetic evaluation in dairy cattle. Under this model, the animals' permanent environment effects are assumed to follow a first-order autoregressive process as long-term (auto-correlations between parities) and short-term (auto-correlations between test-days within lactations) effects, taking into account the nongenetic correlations due to the cows' repeated performance. Currently, given the relevance of genomic prediction in dairy cattle, it is essential to include dense marker information in national genetic evaluations. Therefore, we aimed to evaluate the feasibility of using ssGBLUP methodology to predict GEBV for milk yield of Holstein cattle in Portugal under a multiple-lactation AR model.

\section{MATERIALS AND METHODS}

\section{Data}

A total of 11,434,294 test-day records from the first 3 lactations of Portuguese Holstein cows that calved between 1994 and 2017 were provided by the Portuguese Dairy Cattle Breeders Association (Aveiro, Portugal). The data set was edited according to predefined criteria for genetic analysis with AR models (Carvalheira et al., 2002) and used to calculate genomic predictions (GEBV) using ssGBLUP methodology and traditional EBV for comparisons. The data consisted of 4,725,673 test-day records from 578,552 cows in the first lactation, 3,910,679 test-day records from 486,177 cows in the second lactation, and 2,797,942 test-day records from 353,753 cows in the third lactation. To further test the feasibility of the ssGBLUP methodology, a reduced data set with records truncated at 2012 (excluding records of daughters of young bulls) was also used in the statistical comparisons to evaluate the prediction ability and bias of the new methodology. The reduced data consisted of $3,961,378$ test-day records from 488,811 cows in the first lactation, 3,286,254 testday records from 412,141 cows in the second lactation, and 2,369,824 test-day records from 302,196 cows in the third lactation.
A total of 1,081 genotypes of bulls were provided by the Portuguese Dairy Cattle Breeders Association. Five hundred twenty-five bulls were genotyped using the Illumina BovineSNP50Kv2 BeadChip (Illumina Inc., San Diego, CA) with 54,609 markers. The remaining 556 bulls were genotyped with low-density (GeneSeek Genomic Profiler GGP8K, GGP20K, and GGP25K; Neogen Corp., Lincoln, NE), medium-density (BovineSNP50v1, Illumina Inc.), and high-density (GeneSeek Genomic Profiler GGP80K, and GGP150K; Neogen Corp.) SNP chips. The genotypes of these 556 bulls were imputed to the $50 \mathrm{Kv} 2$ chip using Fimpute 2.2 software (Sargolzaei et al., 2014). Imputation accuracy was estimated based on the Spearman correlation between imputed and known 50Kv2 genotypes. In general, sample accuracies were $>0.96$ for all platforms. Quality control analysis retained SNP and animals with call rates $>0.9$, SNP with minor allele frequency $>0.02$, and lower deviations from Hardy-Weinberg equilibrium $\left(P>1 \times 10^{-6}\right)$. Errors of sex disagreement were checked by heterozygosity on the $\mathrm{X}$ chromosome, and parentprogeny pairs were tested for Mendelian conflict; SNP with unknown position or located on sex chromosomes were not considered in the analyses. Finally, 38,286 autosomal SNP and 1,071 bulls were retained for further analyses. The numbers of genotyped bulls by year of birth are shown in Table 1.

\section{Statistical Modeling}

The AR model for multiple lactations proposed by Carvalheira et al., (2002) was used to implement the traditional genetic (A-AR) and the single-step genomic (H-AR) evaluations for milk yield. In matrix notation, this model may be described as follows:

$$
\mathbf{y}=\mathbf{X} \boldsymbol{\beta}+\mathbf{Z a}+\mathbf{W c}+\mathbf{M p}+\mathbf{Q t}+\mathbf{r},
$$

where $\mathbf{y} \sim N(\mathbf{X} \boldsymbol{\beta}, \mathbf{V})$ is the vector of test-day (TD) records with covariance matrix $\mathbf{V} ; \boldsymbol{\beta}$ is the vector of fixed effects (age class at calving nested within herd, and DIM class nested within herd and lactation); $\mathbf{a} \sim N\left(\mathbf{0}, \mathbf{A} \sigma_{a}^{2}\right)$ is the vector of random animal additive genetic effects, with $\mathbf{A}$ representing the pedigree-based additive genetic relationship matrix or $\mathbf{a} \sim N\left(\mathbf{0}, \mathbf{H} \sigma_{g}^{2}\right)$, in which the combined relationship matrix $\mathbf{H}$ was constructed, including both marker and pedigree information (Aguilar et al., 2010). The variance components $\sigma_{a}^{2}$ and $\sigma_{g}^{2}$ represent the additive genetic variance obtained from the $\mathbf{A}$ and $\mathbf{H}$ matrices, respectively; $\mathbf{c} \sim N(\mathbf{0}, \mathbf{N})$ is the vector of random contemporary group (herd test- 
Table 1. Frequency distribution of genotyped bulls according to the year of birth

\begin{tabular}{|c|c|c|c|c|c|c|}
\hline \multirow[b]{2}{*}{ Birth year } & \multicolumn{6}{|c|}{ Bulls } \\
\hline & All & $\begin{array}{l}\text { With }>9 \\
\text { daughters }\end{array}$ & Young & $\begin{array}{c}\text { No } \\
\text { daughters }\end{array}$ & Validation & $\begin{array}{l}\text { With daughters in } \\
2012 \text { or earlier }\end{array}$ \\
\hline $1966-1975$ & 13 & 4 & - & 2 & - & 11 \\
\hline $1976-1980$ & 34 & 15 & - & 4 & - & 30 \\
\hline 1981-1985 & 43 & 37 & - & - & - & 43 \\
\hline 1986-1990 & 68 & 64 & - & 2 & - & 66 \\
\hline 1991-1995 & 94 & 90 & - & - & - & 94 \\
\hline $1996-2000$ & 99 & 85 & - & 7 & - & 91 \\
\hline 2001-2005 & 164 & 86 & - & 29 & 33 & 93 \\
\hline 2006-2010 & 279 & 46 & - & 91 & 90 & 18 \\
\hline 2011-2012 & 158 & 12 & 107 & - & 11 & - \\
\hline 2013-2015 & 119 & 1 & 118 & - & - & - \\
\hline Total & 1,071 & 440 & 225 & 135 & 134 & 446 \\
\hline
\end{tabular}

date, HTD) effects; $\mathbf{p} \sim N(\mathbf{0}, \mathbf{J})$ is the random longterm environmental effects (LTE); $\mathbf{t} \sim N\left(\mathbf{0}, \mathbf{S}_{L}\right)$ is the random short-term environmental effects (STE), where $L=1,2$, or 3 , corresponding to first, second, and third lactations, respectively; $\mathbf{r} \sim N(\mathbf{0}, \mathbf{R})$ is the vector of random residual effects; and $\mathbf{X}, \mathbf{Z}, \mathbf{W}, \mathbf{M}$, and $\mathbf{Q}$ are incidence matrices relating observations to fixed and random effects. A first-order autoregressive covariance structure was assumed for HTD (within herds), LTE (between parities), and STE (between TD, within lactations) effects. In this context, we have

$$
\mathbf{V}=\mathbf{Z G} \mathbf{Z}^{\prime}+\mathbf{W N W}^{\prime}+\mathbf{M J M}^{\prime}+\sum_{L=1}^{3}\left(\mathbf{Q}_{L} \mathbf{S}_{L} \mathbf{Q}_{L}^{\prime}\right)+\mathbf{R},
$$

where $\mathbf{G}=\mathbf{A} \sigma_{a}^{2}$ for (A-AR) or $\mathbf{G}=\mathbf{H} \sigma_{g}^{2}$ for (H-AR), and $\mathbf{N}, \mathbf{J}$, and $\mathbf{S}_{L}$ are first-order autoregressive covariance structures of the appropriate dimension as follows (for simplicity, let's assume that each herd has 3 HTD levels and cows have $3 \mathrm{TD}$ records in each lactation):

$$
\begin{aligned}
\mathbf{N} & =\sigma_{c}^{2}\left[\begin{array}{ccc}
1 & \rho_{c} & \rho_{c}^{2} \\
& 1 & \rho_{c} \\
s y m & & 1
\end{array}\right] \otimes \mathbf{I}_{q}, \\
\mathbf{J} & =\sigma_{p}^{2}\left[\begin{array}{ccc}
1 & \rho_{p} & \rho_{p}^{2} \\
& 1 & \rho_{p} \\
s y m & & 1
\end{array}\right] \otimes \mathbf{I}_{m}, \\
\mathbf{S}_{L} & =\sigma_{t L}^{2}\left[\begin{array}{ccc}
1 & \rho_{t L} & \rho_{t L}^{2} \\
& 1 & \rho_{t L} \\
s y m & & 1
\end{array}\right] \otimes \mathbf{I}_{m L},
\end{aligned}
$$

and

$$
\mathbf{R}=\mathbf{I}_{n L} \sigma_{e L}^{2}
$$

where $\mathbf{I}$ is the identity matrix; $q$ is the number of herds; $m$ is the number of cows with records; $m L$ is the number of cows within the $L$ th lactation; and $n L$ is the number of records in each lactation; $\sigma_{c}^{2}$ is the HTD variance component, $\rho_{c}$ is the HTD autocorrelation coefficient, $\sigma_{p}^{2}$ is the LTE variance component, $\rho_{p}$ is the LTE autocorrelation coefficient, $\sigma_{t L}^{2}$ is the STE variance component, $\rho_{t L}$ is the STE autocorrelation coefficient, and $\sigma_{e L}^{2}$ is the residual variance components for the records of each lactation. The index $L=1,2$ or 3 corresponds, respectively, to first, second, and third lactations. More details about the autoregressive covariance structure fitted in the AR model may be found in Carvalheira et al. (1998, 2002).

In the $\mathrm{H}-\mathrm{AR}$ model, the inverse of the $\mathbf{H}$ matrix in the mixed model equations is obtained as follows:

$$
\mathbf{H}^{-1}=\mathbf{A}^{-1}+\left[\begin{array}{cc}
0 & 0 \\
0 & \tau\left(0.95 \mathbf{G}+0.05 \mathbf{A}_{22}\right)^{-1}-\omega \mathbf{A}_{22}^{-1}
\end{array}\right],
$$

where $\mathbf{G}$ is the genomic relationship matrix and $\mathbf{A}_{22}$ is the pedigree-based numerator relationship matrix for genotyped animals. The $\mathbf{G}$ matrix was obtained by the first method proposed by VanRaden (2008), in which the allele frequencies were calculated from the current genotyped animals. Weights for $\mathbf{G}(0.95)$ and $\mathbf{A}_{22}(0.05)$ can avoid singularity problems (VanRaden, 2008); $\tau$ is the scaling factor for $\mathbf{G}^{-1}$ and $\omega$ is the scaling factor for $\mathbf{A}_{22}^{-1}$. The $\tau$ value of 1.0 and $\omega$ value of 0.7 were chosen according to Tsuruta et al. (2011). The $\mathbf{H}^{-1}$ matrix was 
calculated using the PreGSF90 software (Misztal et al., 2014).

Preliminary analysis indicated that using the complete data set for variance components estimations using MATLAB (MathWorks Inc., Natick MA) was not practical because of the excessive memory required to store the Cholesky factor of the coefficient matrix of the mixed model equations, necessary to obtain solutions and the log determinant of this matrix in each iteration. For this reason, we opted to build 6 data subsets consisting of 20 herds randomly sampled from the data set with a minimum of 5,000 cows, records spanning across the all period of study, and representing all regions of Portugal to estimate the variance components and autocorrelation coefficients applying derivative free (DF)REML methodology (Smith and Graser, 1986). Likelihood functions were maximized by the multivariate simplex algorithm (Nelder and Mead, 1965) programmed in MATLAB. The convergence criterion was defined as $10^{-8}$. The occurrence of local maxima was checked by running 6 consecutive cold starts without significant changes in the log-likelihood (up to 4 decimal places). This process was repeated for each data subset.

The reliabilities of the breeding values $\left(\mathbf{R}_{e b v}^{2}\right)$ were estimated for each bull as follows:

$$
\mathbf{R}_{e b v}^{2}=1-\frac{P E V_{i}}{\sigma_{a}^{2}\left(1+F_{i}\right)}
$$

where $P E V_{i}$ is the estimated prediction error variance extracted from the diagonal of the inverse of the coefficient matrix for animal $i, 1+F_{i}$ corresponds to the diagonal of the $\mathbf{A}(\mathbf{H})$ matrix for animal $i$, and $\sigma_{a}^{2}\left(\sigma_{g}^{2}\right)$ is the additive genetic variance.

\section{Statistical Analyses}

Rank correlation and differences in reliability among bulls were used to compare the performance of the $\mathrm{A}-\mathrm{AR}$ and $\mathrm{H}-\mathrm{AR}$ models using the complete data sets. To better differentiate the effect of ssGBLUP on the evaluations, these comparisons were done within specific bull categories: genotyped (G0) or nongenotyped (NG0) bulls without daughters; genotyped (G1-9) or nongenotyped (NG1-9) bulls with 1 to 9 daughters; and genotyped (GM9) or nongenotyped (NGM9) bulls with more than 9 daughters.

The predictive ability of the H-AR model was based on the methodology proposed by Mäntysaari et al. (2010). The comparisons were based on the results obtained in each model using different data sets $\left(\mathrm{A}-\mathrm{AR}_{\text {complete }}\right.$ vs. $\mathrm{A}-\mathrm{AR}_{\text {reduced }}$ and $\mathrm{H}-\mathrm{AR}_{\text {complete }}$ vs. $\mathrm{H}-\mathrm{AR}_{\text {reduced }}$ ), where young bulls had no daughter information in the reduced data sets but $>19$ daughters in the complete data sets. Three scenarios were defined for validation of the predictive ability of the H-AR model. In the first scenario (validation 1), 134 genotyped young bulls composed the validation population, where their daughters $(15,256)$ were excluded from the reduced data set. Taking into account that most of these bulls' dams have no daughters or own performance records, the bulls' estimated PA may be biased. To evaluate the effect of a complete pedigree on this estimate, the second scenario (validation 2) assumed the validation population included only 96 genotyped bulls of Portuguese origin, born between 1981 and 2009, in which all their descendants were removed in the reduced data set. For both these scenarios (validations 1 and 2), the data sets included all 1,071 genotyped bulls in the pedigree file. In the third scenario (validation 3), the aim was to evaluate the effect of including noncontributing genotyped bulls (340 genotyped bulls without daughters or relationships in the complete data set). In this scenario, these bulls were excluded from the pedigree file and the 134 young bulls (as in the first scenario) composed the validation population.

To validate the genomic prediction, the DYD computed from the $\mathrm{H}-\mathrm{AR}_{\text {complete }}$ or $\mathrm{A}-\mathrm{AR}_{\text {complete }}$ were regressed on the GEBV (H-AR $\mathrm{Areduced}_{\text {) }}$ or on PA (A$\mathrm{AR}_{\text {reduced }}$ ), respectively. This regression model was as follows (Mäntysaari et al., 2010):

$$
y_{c}=1 b_{0}+b_{1} \hat{a}_{r}+e
$$

where $y_{c}$ is the DYD of the validation bulls calculated in the complete data set (H-AR or A-AR); $b_{0}$ and $b_{1}$ are the intercept and regression coefficients; $\hat{a}_{r}$ is the bull GEBV or PA calculated in the reduced data set; and $e$ is the residual error. The linear regression was weighted by the number of records from their daughters; $b_{1}$ was used as an indicator of bias in the genomic prediction. The empirical reliability $\left(\mathbf{R}_{\text {cor }}\right)$ was measured as follows (Meuwissen et al., 2015):

$$
\mathbf{R}_{c o r}=\operatorname{Corr}(\mathrm{GEBV} \text { or PA, DYD }) / \sqrt{\mathbf{R}_{D Y D}^{2}},
$$

and the DYD reliability $\left(\mathbf{R}_{D Y D}^{2}\right)$ was calculated for each bull as

$$
\mathbf{R}_{D Y D}^{2}=\mathrm{EDC} /(\mathrm{EDC}+K)
$$


where EDC is the effective daughter contribution, in which $\mathrm{EDC}=K \times \mathbf{R}_{e b v}^{2} /\left(1-\mathbf{R}_{e b v}^{2}\right)$ and $K=\left(4-h^{2}\right) / h^{2}$ (Mäntysaari et al., 2010). Differences of $\mathbf{R}_{e b v}^{2}$ between models were also analyzed for each validation test.

Genetic trends for genotyped bulls were estimated by regressing the animals' GEBV and EBV on birth year. Bias of predicted genetic trends was assessed by comparing average GEBV or PA per year from the reduced data set with average GEBV and EBV per year from the complete data set. These evaluations were standardized by deviating all genetic values from the mean EBV of cows born in 2007.

\section{RESULTS}

\section{Genetic Parameters, EBV Reliability, and Rank Correlation}

The variance components, autocorrelations, and heritability estimates for milk yield obtained from the A-AR model are shown in Table 2. These estimates were used in all subsequent analyses. The relative magnitude of these estimates and autocorrelations were consistent with those in the literature (Carvalheira et al., 1998, 2002).

Figure 1A shows the individual reliability $\left(\mathbf{R}_{e b v}^{2}\right)$ obtained from the 2 models (A-AR and H-AR) using the complete data set. In general, the $\mathbf{R}_{e b v}^{2}$ obtained from $\mathrm{H}-\mathrm{AR}$ was always higher. The greatest increase in $\mathbf{R}_{e b v}^{2}$ was observed in genotyped bulls without daughters (G0) and with fewer than 10 daughters (G1-9). For G0

Table 2. Variance components, autocorrelation coefficients, and genetic parameters estimated from traditional evaluation (A-AR model) for milk yield of Portuguese Holstein cattle

\begin{tabular}{lc}
\hline Parameter $^{1}$ & Estimate $\pm \mathrm{SE}$ \\
\hline Additive genetic variance & $10.80 \pm 0.2087$ \\
Error variance for lactation 1 & $7.66 \pm 0.9331$ \\
Error variance for lactation 2 & $10.65 \pm 1.4126$ \\
Error variance for lactation 3 & $11.80 \pm 1.4432$ \\
LTE variance & $<0.001$ \\
LTE autocorrelation & $<0.001$ \\
STE variance for lactation 1 & $11.25 \pm 0.3076$ \\
STE variance for lactation 2 & $23.24 \pm 0.5681$ \\
STE variance for lactation 3 & $28.36 \pm 0.6120$ \\
STE autocorrelation for lactation 1 & $0.83 \pm 0.0036$ \\
STE autocorrelation for lactation 2 & $0.84 \pm 0.0031$ \\
STE autocorrelation for lactation 3 & $0.84 \pm 0.0035$ \\
HTD variance & $4.21 \pm 0.1471$ \\
HTD autocorrelation & $0.51 \pm 0.0230$ \\
Heritability for lactation 1 & $0.32 \pm 0.0102$ \\
Heritability for lactation 2 & $0.22 \pm 0.0076$ \\
Heritability for lactation 3 & $0.20 \pm 0.0001$ \\
\hline
\end{tabular}

${ }^{1} \mathrm{LTE}=$ random long-term environmental effects (between parities); $\mathrm{STE}=$ random short-term environmental effects (between test-days within lactations); HTD = herd test-date. bulls, the mean (SD) and minimum $\mathbf{R}_{e b v}^{2}$ were 0.52 (0.07) and 0.21 for H-AR and only $0.16(0.13)$ and 0.00 for A-AR, respectively. For G1-9 bulls, the mean (SD) and minimum for H-AR were 0.72 (0.09) and 0.44, compared with $0.62(0.16)$ and 0.24 for A-AR, respectively. The difference in $\mathbf{R}_{e b v}^{2}$ for these 2 categories between models was highly significant $(P<0.001)$. For bulls with more than 9 daughters (GM9), the mean $\mathbf{R}_{e b v}^{2}$ was similar among models but its minimum was higher for H-AR than for A-AR (0.82 vs. 0.78). On the other hand, no changes were observed in $\mathbf{R}_{e b v}^{2}$ for nongenotyped bulls (NG1-9 and NGM9). Figure 1B shows the PEV per bull category estimated using the A-AR or H-AR model and the complete data set. For G0, G1-9, and GM9, the PEV was 43,25 , and $8 \%$ lower, respectively, with H-AR than with A-AR. Again, no changes were observed in PEV for nongenotyped bulls across models (NG1-9 and NGM9).

Spearman (rank) correlation coefficients between the animals' genetic merit obtained from $\mathrm{H}-\mathrm{AR}$ and A-AR models (GEBV vs. EBV) across bull categories are given in Table 3. No relevant changes were observed for both models in the rank of nongenotyped bulls or genotyped bulls with $>1$ daughter $(\mathrm{r}>0.90)$. In contrast, we detected significant reranking among the G0 bulls ( $\mathrm{r}$ $=0.61$ ) between the 2 models.

\section{GEBV Validation}

Table 4 shows the average $\mathbf{R}_{e b v}^{2}$ in reduced data sets obtained using the $\mathrm{H}-\mathrm{AR}$ or A-AR models for the 3 validation tests. When young bulls (validation 1 or 3 ) were the validation population, the $\mathbf{R}_{e b v}^{2}$ obtained from the H-AR increased on average by 0.25 compared with that from the A-AR $(P<0.001)$. Similarly, for validation 2 (only Portuguese bulls), the $\mathbf{R}_{e b v}^{2}$ obtained from the $\mathrm{H}-\mathrm{AR}$ increased on average by 0.16 .

The $\mathbf{R}_{\text {cor }}$ obtained for GEBV (H-AR) or EBV (AAR) for the 3 validation tests are shown in Table 4 . For validations 1 and 2, there were no expressive changes in $\mathbf{R}_{\text {cor }}$ between models. In contrast, when noncontributing genotyped bulls were excluded from data sets (validation 3), the $\mathbf{R}_{\text {cor }}$ for H-AR increased on average by 0.08 compared with that for the A-AR model. Removing the noncontributing genotyped bulls (validation 1 vs. validation 3) had no effect on the $\mathbf{R}_{\text {cor }}$ for the A-AR, whereas the H-AR model showed an average improvement of 0.07 . The regression coefficients $\left(b_{1}\right)$, used as an indicator of bias in the genomic prediction, are given in Table 4 . The $b_{1}$ were lower than expected for all validation tests, except when the GEBV or PA from Portuguese bulls was tested (validation 2). The H-AR model was 0.11 and 0.18 less biased than the A-AR 

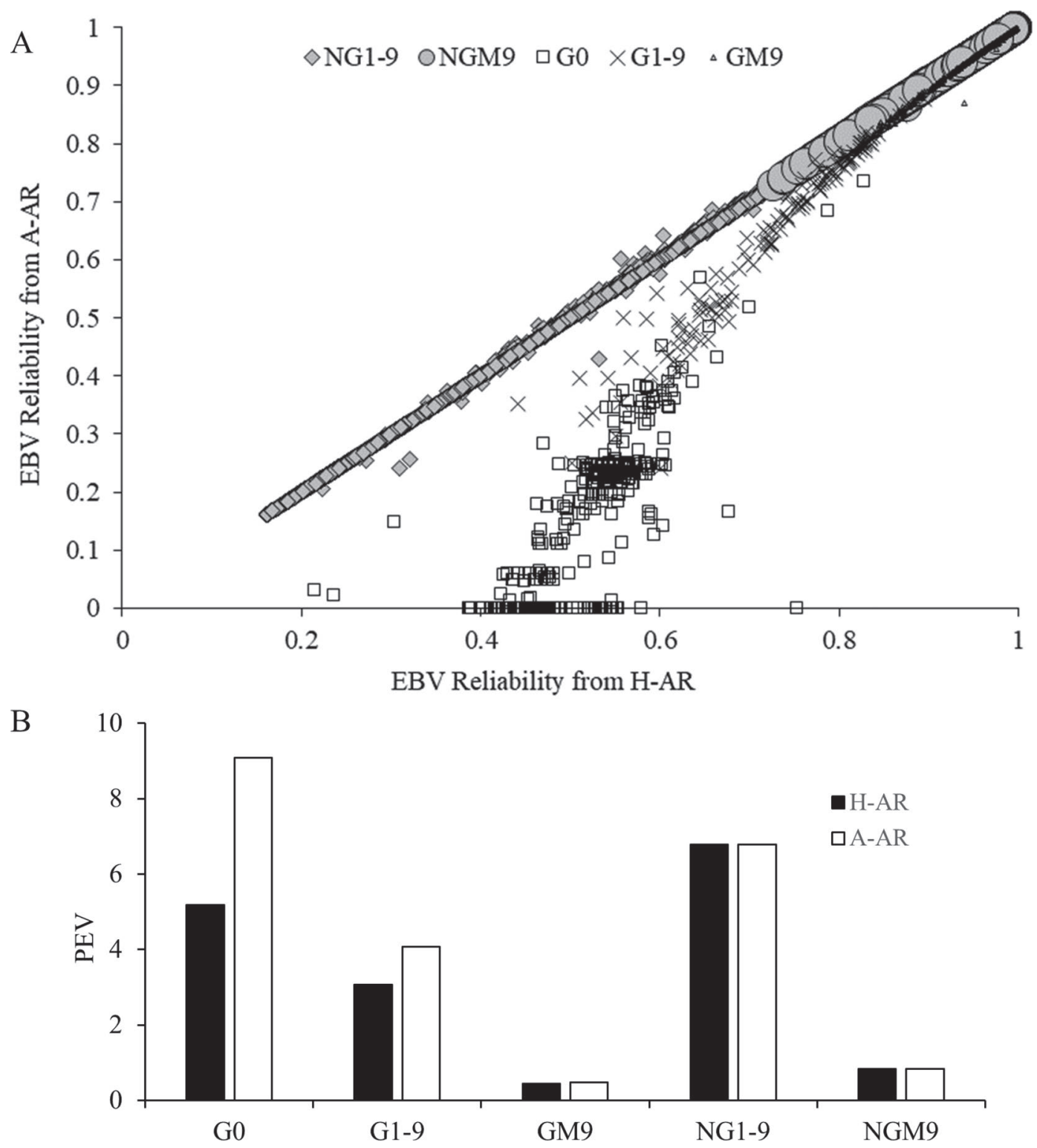

Figure 1. Estimated breeding value reliabilities $\left(\mathbf{R}_{e b v}^{2} ; \mathrm{A}\right)$ and prediction error variance $(\mathrm{PEV} ; \mathrm{B})$ estimated using genomic $(\mathrm{H}-\mathrm{AR})$ and the traditional (A-AR) autoregressive models. G0 = genotvned bulls with no dawohters; G1-9 = genotyped bulls with 1 to 9 daughters; NG1-9= nongenotyped bulls with 1 to 9 daughters; GM9 = ger GEBV Validation n 9 daughters; NGM9= nongenotyped bulls with more than 9 daughters.

model for validations 1 and 3 , respectivel less, for validation 2 , the $b_{1}$ values were $\mathrm{w}$ of the optimal value (Tsuruta et al., 201 models (H-AR and A-AR).

Figure 2 depicts the genetic progress fo between 1985 and 2011, in which the GEBV and EBV were predicted using the $\mathrm{H}-\mathrm{AR}$ and A-AR models with either the complete or reduced data sets. The graphs are arranged to show the progress of all genotyped bulls (Figure 2A, C, and E) versus only genotyped bulls with more than 9 daughters (Figure 2B, D, and F) in each of idation scenarios: validation 1 (panels A and B), ion 2 (panels $\mathrm{C}$ and $\mathrm{D}$ ), and validation 3 (panels F). Except when Portuguese bulls composed the ion test (validation 2; panels $\mathrm{C}$ and $\mathrm{D}$ ), bias in ed genetic trend was observed in all validation tests by visual inspection.

\section{DISCUSSION}

Our study evaluated the feasibility of using the ssGBLUP methodology under an AR model for Portuguese 
Table 3. Number of bulls by category and rank correlation between genomic (G)EBV obtained from the genomic and the traditional autoregressive models

\begin{tabular}{lcc}
\hline Category $^{1}$ & No. of bulls & Rank correlation \\
\hline Genotyped bulls & 360 & \\
G0 & 137 & 0.61 \\
G1-9 & 574 & 0.94 \\
GM9 & & 0.99 \\
Nongenotyped bulls & 5,000 & 0.98 \\
NG0 & 21,317 & 0.99 \\
NG1-9 & 4,420 & 0.99 \\
NGM9 & \\
\hline
\end{tabular}

${ }^{1}$ Genotyped (G0) or nongenotyped (NG0) bulls with no daughters; genotyped (G1-9) or nongenotyped (NG1-9) bulls with 1 to 9 daughters; genotyped (GM9) or nongenotyped (NGM9) bulls with more than 9 daughters.

Holstein cattle, taking into account the relatively small number of genotyped bulls available. Comparisons were based on differences between individual EBV reliabilities, rank correlation, predictive ability, and genetic trends.

The additive genetic relationship between the animals affected the individual reliability of genomic predictions, particularly in genotyped bulls with few or no daughters. The improvement of the $\mathbf{R}_{e b v}^{2}$ ranged from 0.10 to 0.36 when including genomic information in the analysis (Figure 1A), a direct consequence of the reduction (up to $43 \%$ ) in PEV (Figure 1B). This finding is supported by previous studies in which the genomic evaluation was more beneficial for young animals with low accuracy on a traditional EBV evaluation (Hayes et al., 2009; VanRaden et al., 2009). Similar results were observed by Forni et al. (2011) and Oh et al. (2017). These authors also used a small number of genotyped animals in the population (e.g., 1,038 and 1,989 pigs, respectively) and, as in the present study, found higher estimates of reliabilities only for genotyped animals. In contrast, Guo et al. (2015), using 3,445 genotyped ani- mals, reported an effective improvement in reliabilities for nongenotyped animals. Moreover, in our study, we observed significant reranking among bulls with no daughters $(r=0.61)$ when genomic information was included (Table 3 ).

In terms of predictive ability, the inclusion of genomic information improved $\mathbf{R}_{e b v}^{2}$ from 0.16 to 0.26 for all validation scenarios (Table 4). Similarly, the empirical reliability $\left(\mathbf{R}_{\text {cor }}\right.$ ) increased up to 0.08 (Table 4). As a measure of accuracy, our results of $\mathbf{R}_{\text {cor }}$ were in general lower than in other reports (e.g., Koivula et al., 2015; Baba et al., 2017). Using ssGBLUP, both the Koivula and Baba papers included more than 5,000 genotyped animals in their study and found reliabilities ranging from 0.12 to 0.24 . VanRaden et al. (2009) showed that reliability values increased linearly with the number of genotyped bulls under GBLUP. Although ssGBLUP is more versatile due to the incorporation of phenotypes of nongenotyped relatives of the selection candidates, the number of genotyped bulls seems to affect the reliability values. Also, the work of Lourenco et al. (2014), which used ssGBLUP in a population with a relatively small number of genotyped animals $(1,648)$, found an increase in reliability of 0.06 , similar to results of the present study. In the study of Clark et al. (2012), the authors showed that close relationships between the animals in the validation and reference populations resulted in higher reliability for GEBV. In this context, considerable gains in reliability may also be expected with ssGBLUP with a relatively small number of genotyped animals, because there is a closer relationship between reference and predicted animals. The average relationship within the reference population may also affect the average reliability, as reported by Pszczola et al. (2012). Those authors showed that when the animals in the reference population were related to each other, the reliabilities were also higher, implying that the design of the reference population is

Table 4. Mean (SD) of EBV reliabilities $\left(\mathbf{R}_{e b v}^{2}\right)$, empirical reliabilities $\left(\mathbf{R}_{c o r}\right)$, and regression coefficients $\left(b_{1}\right)$ for 3 validation tests, obtained from the genomic $(\mathrm{H}-\mathrm{AR})$ and traditional $(\mathrm{A}-\mathrm{AR})$ autoregressive models using the reduced data set ${ }^{1}$

\begin{tabular}{|c|c|c|c|c|c|c|}
\hline \multirow[b]{2}{*}{ Test $^{2}$} & \multicolumn{3}{|c|}{ H-AR } & \multicolumn{3}{|c|}{ A-AR } \\
\hline & $\mathbf{R}_{e b v}^{2}$ & $\mathbf{R}_{c o r}$ & $b_{1}$ & $\mathbf{R}_{e b v}^{2}$ & $\mathbf{R}_{c o r}$ & $b_{1}$ \\
\hline Validation 1 & $0.52(0.06)$ & 0.17 & 0.36 & $0.26(0.10)$ & 0.16 & 0.25 \\
\hline Validation 2 & $0.52(0.06)$ & 0.82 & 1.15 & $0.36(0.11)$ & 0.79 & 0.99 \\
\hline Validation 3 & $0.51(0.06)$ & 0.24 & 0.43 & $0.26(0.10)$ & 0.16 & 0.25 \\
\hline \multicolumn{7}{|c|}{$\begin{array}{l}{ }^{1} \mathrm{R}_{\text {cor }}\left(b_{1}\right) \text { were correlated (regressed) with daughter yield deviations obtained from the respective models using } \\
\text { the complete data set. }\end{array}$} \\
\hline \multicolumn{7}{|c|}{$\begin{array}{l}{ }^{2} \text { Validation } 1=1,071 \text { genotyped bulls included in pedigree and } 134 \text { younger bulls in validation test; validation } \\
2=1,071 \text { genotyped bulls included in pedigree and } 96 \text { Portuguese bulls in validation test; validation } 3=73 \\
\text { genotyped bulls included in pedigree (noncontributing genotyped bulls excluded) and } 134 \text { younger bulls in } \\
\text { validation test. }\end{array}$} \\
\hline
\end{tabular}


also important when genotyped and nongenotyped animals are jointly evaluated using the $\mathbf{H}$ matrix.

In agreement with the study of Li et al. (2014), in our study, the estimated average $\mathbf{R}_{e b v}^{2}$ were much higher than $\mathbf{R}_{\text {cor }}$ when young bulls were the validation test population (Table 4). This result occurs because the bulls selected for the validation tests and selection itself reduce the additive genetic variance and therefore the empirical reliability of selection. This is consistent with previous studies (Bijma, 2012; Gorjanc et al., 2015), which showed that prediction accuracies or predictive ability are biased downward by selection.

The regression coefficients measuring bias from validation tests in young bulls were less than 1.0 (Table 4). A possible reason could be that $85.5 \%$ of the bulls were foreign and only $9.8 \%$ of the genotyped bulls were
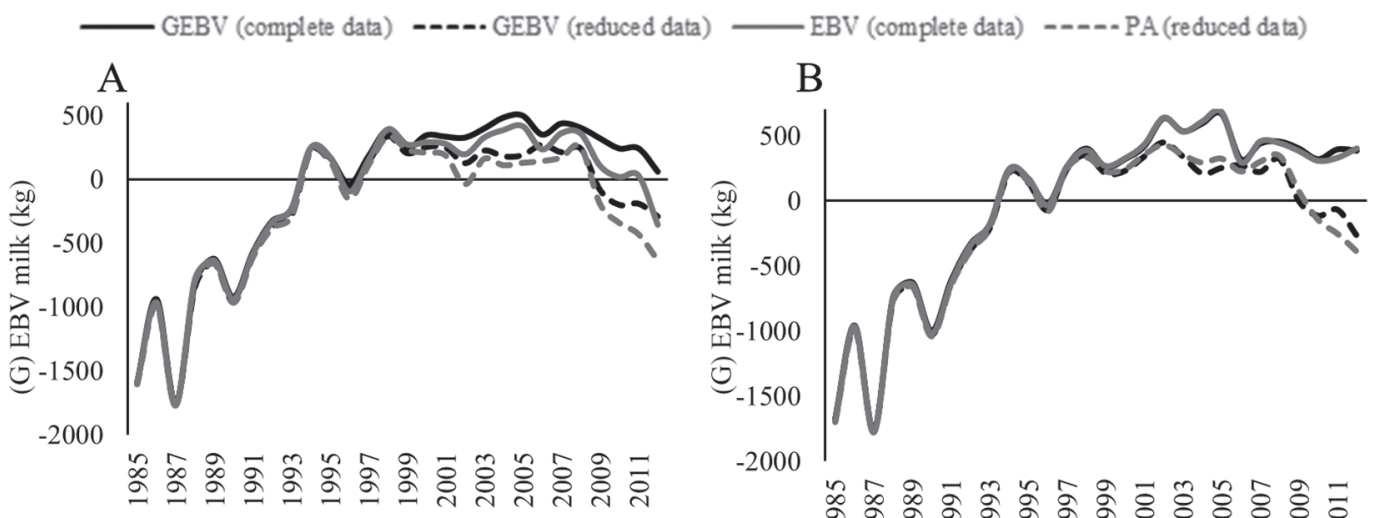

ڤ
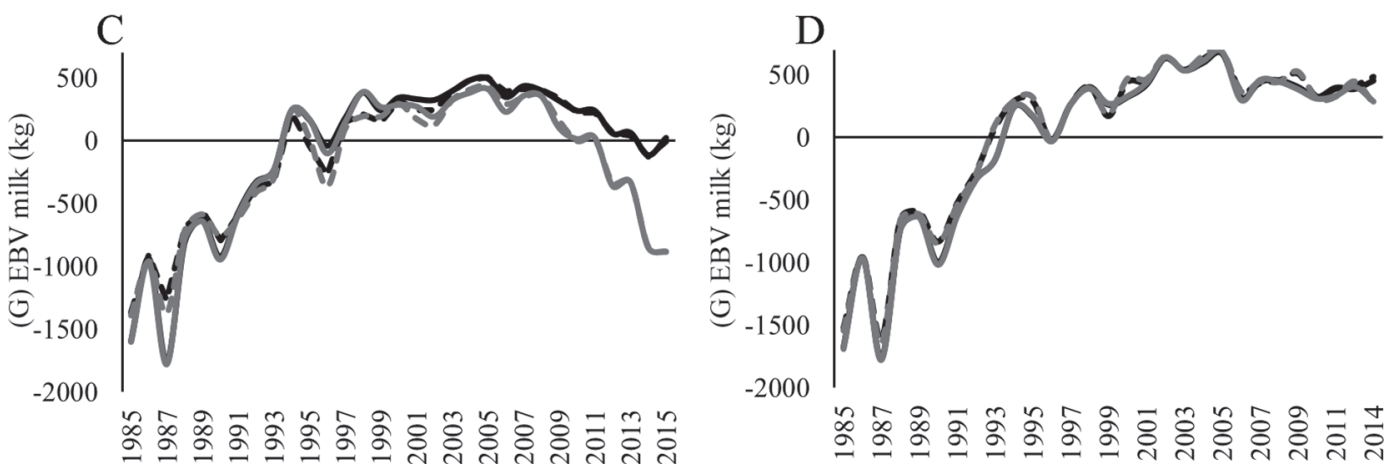

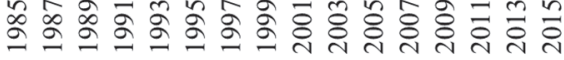
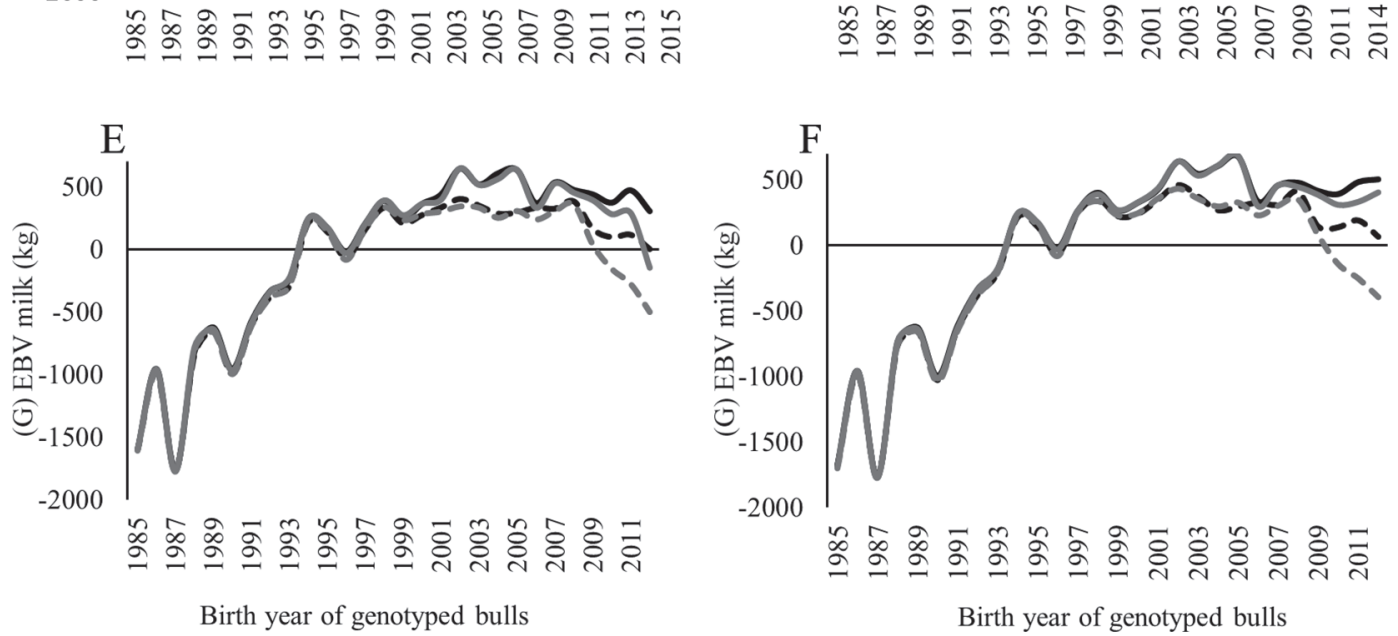

Figure 2. Bull genetic trends for the 3 scenarios of validation tests. The panels on the left (A, C, and E) show the genetic trends for all bulls (genotyped or not) for each validation test. The panels on the right (B, D, and F) show the genetic trends for bulls with more than 9 daughters (genotyped or not) for each validation test. Validation 1 (panels A and B) = 134 younger bulls in validation test; validation 2 (panels C and D) $=96$ Portuguese bulls in validation test; validation 3 (panels $\mathrm{E}$ and $\mathrm{F}$ ) $=134$ younger bulls in validation test but excluding 340 noncontributing genotyped bulls from the pedigree. GEBV = genomic breeding value; $\mathrm{PA}=$ parent average. 
from the Portuguese population. This implies that most of bull dams had no daughters or own phenotypic performance records, which may have induced bias on the PA estimation for young bulls. A similar situation was reported by Pribyl et al. (2014) in which, for a small Holstein population, the majority of the AI were made with imported semen with bulls having low or only indirect genetic relationships with the population. This interpretation is in line with our results on the validation tests for the Portuguese bulls where the prediction bias was within an acceptable range.

Bias on predicted genetic trends was consistent with the interpretation of bias from the regression analysis (Figure 2). The reason for the bias on the genetic trend can be explained in part by the fact that the reference animals did not have all the information required to trace selection, especially on bull dams, because most of them are from foreign origin with no other source of information. Similar to reports by Ma et al. (2015) and Baba et al. (2017), the bias found on GEBV prediction was lower than in PA. Ma et al. (2015) evaluated several strategies to reduce bias on genetic trend and concluded that the most efficient way is to implement a single-step approach. In addition, the superiority of $\mathrm{H}-\mathrm{AR}$ over A-AR is well depicted in the genetic progress when unproven genotyped bulls (bulls with 1 to 9 daughters) were included (Figure 2).

We hypothesized that the inclusion of noncontributing genotyped bulls (with no reliability or no daughters in the traditional evaluation) in the pedigree would contribute by identical-by-state (IBS) to improve the relationship matrix and, therefore, to the reliability of the genomic prediction (13\% of the bulls were from an older generation with the remainder being young bulls). In the traditional evaluation, the inclusion of those bulls has no effect on predictive ability or bias because of the almost total lack of relationship with the rest of the population. On the other hand, in the genomic prediction, the inclusion of these bulls was more beneficial for themselves compared with the traditional evaluation (Figure 1), improving their individual $\mathbf{R}_{e b v}^{2}$ and having only a minor effect on predictive ability. Previous studies showed that when relatives are in the reference population, the importance of information on distantly related animals might be reduced (Clark et al., 2012; Pocrnic et al., 2017).

In the present study, we analyzed the feasibility of using the ssGBLUP approach with an AR model with a relatively small number of genotyped bulls. The H-AR model provided higher individual EBV reliabilities for young bulls. In terms of predictive ability, bias occurred mostly due to the lack of phenotypic information on bull dams (foreign origin). In general, H-AR promoted higher average EBV and better empirical reliabilities and reduced bias compared with traditional evaluations.

\section{CONCLUSIONS}

These results suggest that the ssGBLUP methodology applied to AR models is feasible and may be advantageous to the Portuguese national genetic evaluations. With the anticipated increase in the number of genotyped animals (e.g., by including females), it is expected that the $\mathrm{H}-\mathrm{AR}$ will provide even higher reliabilities, especially for young stock, thus contributing to the improvement of the genetic progress of the Portuguese dairy cattle population.

\section{ACKNOWLEDGMENTS}

The authors acknowledge the Portuguese Dairy Cattle Breeders Association (Aveiro, Portugal) and Embrapa Dairy Cattle (Juiz de Fora, Brazil) for providing data for this study. This study was partially financed by Coordination for the Improvement of Higher Education Personnel and Portuguese National Funding Agency for Science, Research and Technology (CAPES/FCT, no. 99999.008462/2014-03 and 88887.136171/2017-00), and National Council of Technological and Scientific Development (CNPq 465377/2014-9; PROGRAMA INCT).

\section{REFERENCES}

Aguilar, I., I. Misztal, D. L. Johnson, A. Legarra, S. Tsuruta, and T. J. Lawlor. 2010. Hot topic: A unified approach to utilize phenotypic, full pedigree, and genomic information for genetic evaluation of Holstein final score. J. Dairy Sci. 93:743-752. https://doi.org/10 $.3168 /$ jds.2009-2730.

Baba, T., Y. Gotoh, S. Yamaguchi, S. Nakagawa, H. Abe, Y. Masuda, and T. Kawahara. 2017. Application of single-step genomic best linear unbiased prediction with a multiple-lactation random regression test-day model for Japanese Holsteins. Anim. Sci. J. 88:1226-1231. https://doi.org/10.1111/asj.12760.

Bijma, P. 2012. Accuracies of estimated breeding values from ordinary genetic evaluations do not reflect the correlation between true and estimated breeding values in selected populations. J. Anim. Breed. Genet. 129:345-358. https://doi.org/10.1111/j.1439-0388 .2012.00991.x.

Carvalheira, J., E. J. Pollak, R. L. Quaas, and R. W. Blake. 2002. An autoregressive repeatability animal model for test-day records in multiple lactations. J. Dairy Sci. 85:2040-2045. https://doi.org/10 $.3168 /$ jds.S0022-0302(02)74281-1.

Carvalheira, J. G., R. W. Blake, E. J. Pollak, R. L. Quaas, and C. V. Duran-Castro. 1998. Application of an autoregressive process to estimate genetic parameters and breeding values for daily milk yield in a tropical herd of Lucerna cattle and in United States Holstein herds. J. Dairy Sci. 81:2738-2751. https://doi.org/10.3168/ jds.S0022-0302(98)75831-X.

Christensen, O. F., and M. S. Lund. 2010. Genomic prediction when some animals are not genotyped. Genet. Sel. Evol. 42:2. https:// doi.org/10.1186/1297-9686-42-2. 
Clark, S. A., J. M. Hickey, H. D. Daetwyler, and J. H. J. van der Werf. 2012. The importance of information on relatives for the prediction of genomic breeding values and the implications for the makeup of reference data sets in livestock breeding schemes. Genet. Sel. Evol. 44:4. https://doi.org/10.1186/1297-9686-44-4.

Forni, S., I. Aguilar, and I. Misztal. 2011. Different genomic relationship matrices for single-step analysis using phenotypic, pedigree and genomic information. Genet. Sel. Evol. 43:1-7. https://doi .org/10.1186/1297-9686-43-1.

Gorjanc, G., P. Bijma, and J. M. Hickey. 2015. Reliability of pedigreebased and genomic evaluations in selected populations. Genet. Sel. Evol. 47. https://doi.org/10.1186/s12711-015-0145-1.

Guo, X., O. F. Christensen, T. Ostersen, Y. Wang, M. S. Lund, and G. Su. 2015. Improving genetic evaluation of litter size and piglet mortality for both genotyped and nongenotyped individuals using a single-step method. J. Anim. Sci. 93:503-512. https://doi.org/10 $.2527 /$ jas.2014-8331.

Hayes, B. J., P. J. Bowman, A. J. Chamberlain, and M. E. Goddard. 2009. Invited review: Genomic selection in dairy cattle: Progress and challenges. J. Dairy Sci. 92:433-443. https://doi.org/10.3168/ jds.2008-1646.

Jattawa, D., M. A. Elzo, S. Koonawootrittriron, and T. Suwanasopee. 2016. Genomic-polygenic and polygenic evaluations for milk yield and fat percentage using random regression models with Legendre polynomials in a Thai multibreed dairy population. Livest. Sci. 188:133-141. https://doi.org/10.1016/j.livsci.2016.04.019.

Koivula, M., I. Strandén, J. Pösö, G. P. Aamand, and E. A. Mäntysaari. 2015. Single-step genomic evaluation using multitrait random regression model and test-day data. J. Dairy Sci. 98:2775-2784. https://doi.org/10.3168/jds.2014-8975.

Li, X., S. Wang, J. Huang, L. Li, Q. Zhang, and X. Ding. 2014. Improving the accuracy of genomic prediction in Chinese Holstein cattle by using one-step blending. Genet. Sel. Evol. 46:66. https:/ /doi.org/10.1186/s12711-014-0066-4.

Lourenco, D. A. L., I. Misztal, S. Tsuruta, I. Aguilar, E. Ezra, M. Ron, A. Shirak, and J. I. Weller. 2014. Methods for genomic evaluation of a relatively small genotyped dairy population and effect of genotyped cow information in multiparity analyses. J. Dairy Sci. 97:1742-1752. https://doi.org/10.3168/jds.2013-6916.

Ma, P., M. S. Lund, U. S. Nielsen, G. P. Aamand, and G. Su. 2015. Single-step genomic model improved reliability and reduced the bias of genomic predictions in Danish Jersey. J. Dairy Sci. 98:9026-9034. https://doi.org/10.3168/jds.2015-9703.

Mäntysaari, E., Z. Liu, and P. VanRaden. 2010. Interbull validation test for genomic evaluations. Interbull Bull. 41:17-22.

Meuwissen, T. H. E., M. Svendsen, T. Solberg, and J. Ødegård. 2015. Genomic predictions based on animal models using genotype imputation on a national scale in Norwegian Red cattle. Genet. Sel. Evol. 47:79. https://doi.org/10.1186/s12711-015-0159-8.
Misztal, I., A. Legarra, and I. Aguilar. 2009. Computing procedures for genetic evaluation including phenotypic, full pedigree, and genomic information. J. Dairy Sci. 92:4648-4655. https://doi.org/10 3168/jds.2009-2064.

Misztal, I., S. Tsuruta, D. A. L. Lourenco, I. Aguilar, A. Legarra, and Z. G. Vitezica. 2014. Manual for BLUPF90 family of programs. http://nce.ads.uga.edu/wiki/doku.php?id=application_programs.

Nelder, J. A., and R. Mead. 1965. A simplex method for function minimization. Comput. J. 7:308-313. https://doi.org/10.1093/comjnl/ 7.4.308

Oh, J. D., C. S. Na, and K. Do Park. 2017. Validation of selection accuracy for the total number of piglets born in Landrace pigs using genomic selection. Asian-australas. J. Anim. Sci. 30:149-153. https://doi.org/10.5713/ajas.16.0394.

Pocrnic, I., D. A. L. Lourenco, H. L. Bradford, C. Y. Chen, and I. Misztal. 2017. Technical note: Impact of pedigree depth on convergence of single-step genomic BLUP in a purebred swine population. J. Anim. Sci. 95:3391-3395. https://doi.org/10.2527/jas.2017 .1581

Pribyl, J., J. Bauer, P. Pesek, J. Pribylová, L. Vostrý, and L. Zavadilová. 2014. Domestic and Interbull information in the single step genomic evaluation of Holstein milk production. Czech J. Anim. Sci. 9:409-415.

Pszczola, M., T. Strabel, J. A. M. van Arendonk, and M. P. L. Calus. 2012. The impact of genotyping different groups of animals on accuracy when moving from traditional to genomic selection. J. Dairy Sci. 95:5412-5421. https://doi.org/10.3168/jds.2012-5550.

Sargolzaei, M., J. P. Chesnais, and F. S. Schenkel. 2014. A new approach for efficient genotype imputation using information from relatives. BMC Genomics 15:478. https://doi.org/10.1186/1471 $-2164-15-478$.

Smith, S. P., and H.-U. Graser. 1986. Estimating variance components in a class of mixed models by restricted maximum likelihood. J. Dairy Sci. 69:1156-1165. https://doi.org/10.3168/jds.S0022 -0302(86)80516-1.

Tsuruta, S., I. Misztal, I. Aguilar, and T. J. Lawlor. 2011. Multiple-trait genomic evaluation of linear type traits using genomic and phenotypic data in US Holsteins. J. Dairy Sci. 94:4198-4204. https://doi.org/10.3168/jds.2011-4256.

VanRaden, P. M. 2008. Efficient methods to compute genomic predictions. J. Dairy Sci. 91:4414-4423. https://doi.org/10.3168/jds .2007-0980.

VanRaden, P. M., C. P. Van Tassell, G. R. Wiggans, T. S. Sonstegard, R. D. Schnabel, J. F. Taylor, and F. S. Schenkel. 2009. Invited review: Reliability of genomic predictions for North American Holstein bulls. J. Dairy Sci. 92:16-24. https://doi.org/10.3168/jds .2008-1514. 